\title{
Supplementary Information \\ Polar nanodomains in a ferroelectric superconductor
}

Salva Salmani-Rezaie, Kaveh Ahadi, and Susanne Stemmer

Materials Department, University of California, Santa Barbara, California 93106-5050, USA

\section{Polar domains in undoped $\mathrm{SrTiO}_{3}$}

Figure S1 shows polar nanodomains in the paraelectric (a) and ferroelectric (b) phase of an undoped, strained $\mathrm{SrTiO}_{3}$ film on LSAT, see ref. [1] for more details and images. Images of polar domains above the Curie temperature support an order-disorder transition. Upon approaching the ferroelectric transition temperature, polar nanodomains reorient and percolate to form a single domain ferroelectric phase with the polarization direction of normal to the film plane [2].
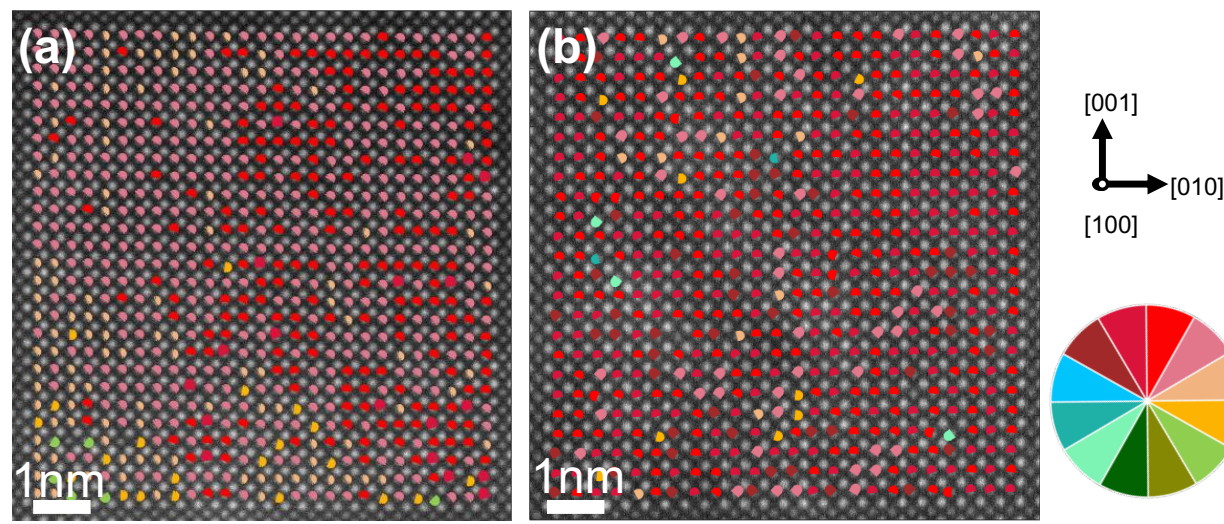

Figure S-1: Polar nanodomains in the (a) paraelectric and (b) ferroelectric phase of an undoped strained $\mathrm{SrTiO}_{3}$ film.

\section{Nanodomains across the film thickness}

The $\mathrm{SrTiO}_{3}$ films are uniformly strained. HAADF-STEM images were obtained from cross-section samples across the entire film thickness. Figure S2 shows a region close to the film surface (a and b) and a region close to the interface ( $\mathrm{c}$ and $\mathrm{d}$ ), respectively, from the film with $6 \times 10^{19} \mathrm{~cm}^{-3}$ carrier density. The TEM foil thickness increases from the film surface toward the interface. Position averaged convergent beam diffraction (PACBED) patterns (b and d) reflect the change in thickness of the TEM foil [3]. Regardless of the local TEM foil thickness at the region analyzed, the nanodomains are present. 

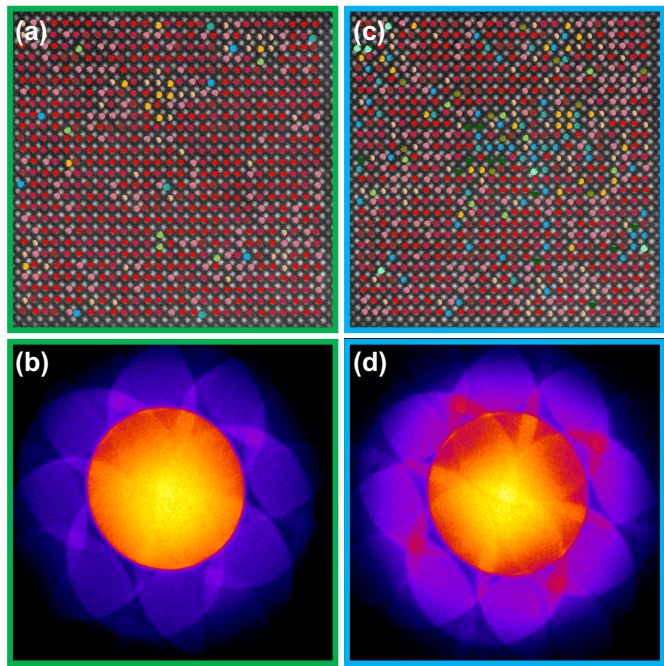

Figure S2: Nanodomain formation across the film thickness

\section{Statistical distribution of the Ti-O Column displacements}

We analyzed more than 7,000 Ti-O columns for strained and unstrained $\mathrm{SrTiO}_{3}$ films with different carrier densities (see main text). Figure S3 shows the statistical distributions of the displacement values. Nanodomains do not form in the undoped, unstrained $\mathrm{SrTiO}_{3}$ films [1] and the average Ti-O column displacement is very small $(5.6 \mathrm{pm})$, which serves a measure of the experimental error. Undoped, strained $\mathrm{SrTiO}_{3}$ films have the largest average polar distortions $(10.9 \mathrm{pm})$ associated with large polar domains and a high Curie temperature. Increasing the dopant concentration suppresses the average until the displacements due to the dopant atoms themselves affect the distributions. For this reason, we see an upturn in the average in the over-doped sample $\left(n_{3 \mathrm{D}}=3 \times 10^{20} \mathrm{~cm}^{-3}\right)$. At large dopant concentrations the number of columns with very large displacements in the tail of distribution increases.

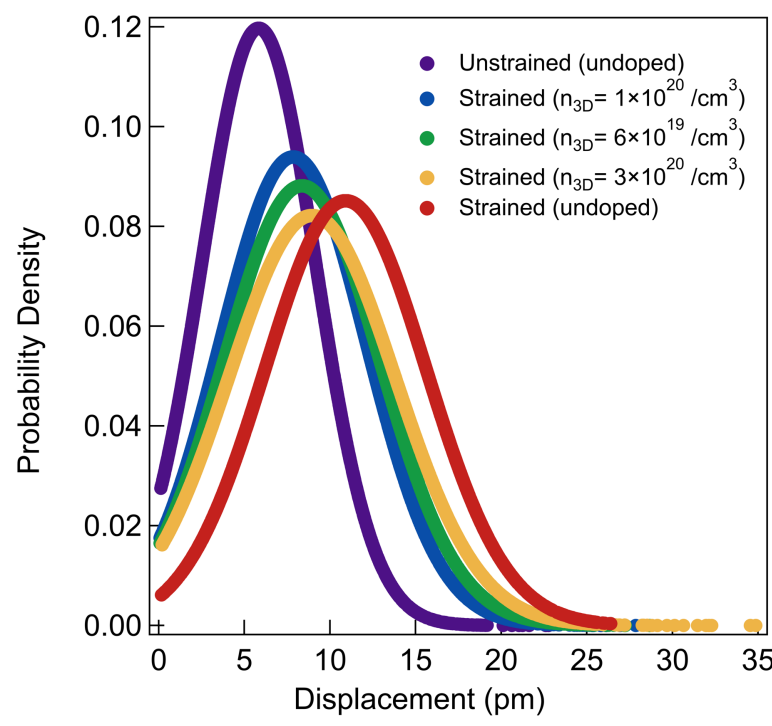

Figure S3: Distribution of the Ti-O column displacements at room temperature. 


\section{Superconducting transition temperatures}

Figure S4 shows the superconducting critical temperatures, taken from ref. [4], as a function of the average magnitude of the displacements taken from Fig. S1. The critical temperature is inversely proportional to the average magnitude of polar distortions and the peak of the superconductivity dome corresponds to the smallest polar displacement. The samples with nanodomains $\left(n_{3 \mathrm{D}}=6 \times 10^{19} \mathrm{~cm}^{-3}\right.$ and $\left.n_{3 \mathrm{D}}=1 \times 10^{20} \mathrm{~cm}^{-3}\right)$ show a doubling in critical temperature of superconductivity, relative to the values of unstrained $\mathrm{SrTiO}_{3}$ [4]. It is important to note that superconducting dome as a function of carrier density in the strained films follows the same qualitative trend as bulk, unstrained $\mathrm{SrTiO}_{3}$, for which the maximum superconducting temperature also occurs around $1 \times 10^{20} \mathrm{~cm}^{-3}$.

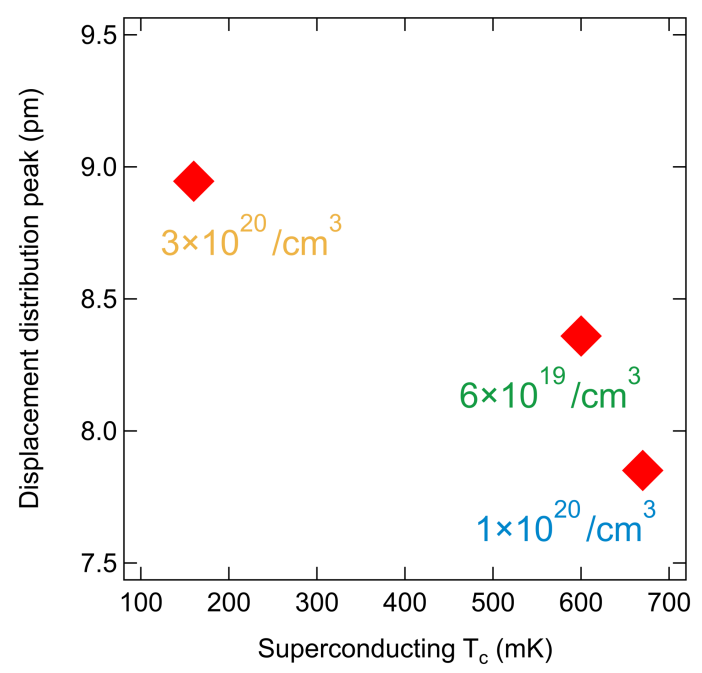

Figure S4: Superconducting critical temperature as a function of the average magnitude of the displacement.

\section{References}

[1] S. Salmani-Rezaie, K. Ahadi, W. M. Strickland, and S. Stemmer, Phys. Rev. Lett. (submitted).

[2] R. Russell, N. Ratcliff, K. Ahadi, L. Y. Dong, S. Stemmer, and J. W. Harter, Phys. Rev. Mater. 3, 091401 (2019).

[3] J. M. LeBeau, S. D. Findlay, L. J. Allen, and S. Stemmer, Ultramicroscopy 110, 118 (2010).

[4] K. Ahadi, L. Galletti, Y. Li, S. Salmani-Rezaie, W. Wu, and S. Stemmer, Sci. Adv. 5, eaaw0120 (2019). 